\title{
THE ZEROS OF $P_{n_{\imath}}^{\prime}\left(x_{0}\right)$ OF NON-INTEGRAL DEGREE
}

\author{
K M. Siegeu, J. W. Crispin, R. E. Kleinman and H. E. Hunter
}

1. Introduction. In January, 1951, tables were published [1] on the zeros of $P_{n_{2}}^{\prime}(x)$, the Associated Legendre Function of order 1 and non-integral degree. In Aprll, 1951, certain errors were observed [2] in those tables of Reference 1.

This note explains a method devised for use at the Willow Run Research Center which, though not as accurate as the method described in Reference 2, can still yield results suitable for engineering problems. Throughout we will consider the general problem for $n_{\imath}$ real and $x_{0}=\cos \theta_{0}$. We will continually use as a numerical example $x_{0}=\cos 165^{\circ}$.

We are interested in finding the values of $n_{\imath}$ and $\int_{x_{0}}^{1}\left[P_{n_{2}}^{\prime}(x)\right]^{2} d x$ where the $n_{\imath}$ 's are such that

$$
P_{n_{1}}^{\prime}\left(x_{0}\right)=0
$$

In order to introduce as many bounds and checks of results as possible, the following asymptotic relations and observations were employed.

When the $n_{\imath}$ are large, we obtain from the asymptotic formula [3]:

$$
n_{\imath+1}-n_{\imath}=\pi / \theta_{0}
$$

The two limiting cases for flow around a cone or scattering by a cone are $\theta_{0}=\pi$ and $\theta_{0}=\pi / 2$, and for these two limiting cases the $n_{\imath}$ differ by 1 and 2 , respectively.

Thus for $\theta_{0}=165^{\circ}$ the maximum value of the differences between successive $n_{\imath}$ 's is 1.0909. A few asymptotic difference values are shown in Table 1.

In the $90^{\circ}$ and $180^{\circ}$ cases we wll now show that the asymptotic forms are the exact forms for all $\imath$.

Consider

$$
P_{n_{\imath}}^{m}(0)=\frac{\sqrt{\pi} 2^{m}}{\Gamma\left[\frac{1}{2}\left(n_{\imath}-m\right)+1\right] \Gamma\left[\frac{1}{2}-\frac{1}{2}\left(n_{\imath}+m\right)\right]}
$$

Substituting $m=1$, the case under discussion, one obtains

$$
P_{n_{\imath}}^{\prime}(0)=\frac{2 \sqrt{\pi}}{\Gamma\left(\frac{1}{2} n_{\imath}+\frac{1}{2}\right) \Gamma\left(-\frac{1}{2} n_{\imath}\right)} .
$$

Since $P_{n_{2}}^{m}(x)=P_{-n_{2}-1}^{m}(x)$ (Ref. 4, p 62) in order not to introduce degenerate forms we will find the critical value of $n_{\imath}$ and only consider $n_{\imath}$ greater than this critical value.

Thus

$$
n_{\imath}^{c}=-n_{\imath}^{c}-1 \text { i.e., } n_{i}^{c}=-\frac{1}{2}
$$

Therefore, we will only consider those $n_{i}$ 's such that

$$
n_{\imath}>-\frac{1}{2}
$$


By (1.1) and (1.2) this would mean, when $\theta_{0}=\pi / 2$, that

$$
\frac{1}{\Gamma\left(\frac{1}{2} n_{\imath}+\frac{1}{2}\right) \Gamma\left(-\frac{1}{2} n_{\imath}\right)}=0
$$

Thus, either $\frac{1}{2}\left(n_{\imath}+1\right)$ or $-\frac{1}{2} n_{\imath}$ must be poles of the gamma function $\Gamma\left(\frac{1}{2} n_{\imath}+\frac{1}{2}\right)$ has no poles for $n_{\imath}>-\frac{1}{2}$, but $\Gamma\left(-\frac{1}{2} n_{\imath}\right)$ has poles for $n_{\imath}=0,2,4,6, \cdots$.

Therefore

$$
n_{\imath}=2 i \quad i \geqq 0
$$

In the case of $\theta_{0}=180^{\circ}$

$$
P_{n_{\imath}}^{\prime}(x)=-\frac{1}{2} n_{\imath}\left(n_{\imath}+1\right)\left(1-x^{2}\right)^{\frac{1}{2}}{ }_{2} F_{1}\left[1-n_{\imath}, n_{\imath}+2 ; 2 ; \frac{1}{2}(1-x)\right]
$$

(Ref. 4, p. 63)

TABLE 1

\begin{tabular}{r|r}
$\theta_{0}$ & $\pi / \theta_{0}$ \\
\hline $90^{\circ}$ & 2.0000 \\
$165^{\circ}$ & 1.0909 \\
$170^{\circ}$ & 1.0588 \\
$175^{\circ}$ & 10286 \\
$180^{\circ}$ & 10000 \\
\hline
\end{tabular}

We observe as $x \rightarrow-1$ that the right hand side of (1.5) becomes indeterminate; thus, to evaluate it at this point we must determine

$$
\begin{aligned}
& \lim _{z=(\pi-\theta) \rightarrow+0} P_{n_{\imath}}^{\prime}(\cos \theta) \\
& \quad=-\frac{1}{2} n_{\imath}\left(n_{\imath}+1\right) z_{2} F_{1}\left(1-n_{\imath}, n_{\imath}+2,2 ;\left\{1-\frac{1}{4} z^{2}+0\left(z^{4}\right)\right\}\right)
\end{aligned}
$$

Since the ${ }_{2} F_{1}$ diverges for $x=-1 \mathrm{ln}$ (1.5), and since we can expand (1.6) in a Maclaurin series in $z$, and since $z$ times it also drverges, then a priori, (1.6) diverges. In order that (1.6) be consistent with $(1.1),{ }_{2} F_{1}\left[1-n_{2}, n_{\imath}+2 ; 2 ; \frac{1}{2}(1-\mathrm{x})\right]$ must cut off. Hence, either $\left(1-n_{2}\right)$ or $\left(n_{2}+2\right)$ should be zero or a negative integer. By (1.3), $\left(n_{\imath}+2\right)$ is always $>3 / 2$; thus, $\left(1-n_{\imath}\right)$ must equal zero or a negative integer, i.e.,

$$
n_{2}=\imath \quad i=1,2,3, \cdots \text {. }
$$

Thus, we observe that the asymptotic form for the differences (Table 1 ) is the exact form for all $\left(n_{\imath+1}-n_{\imath}\right)$ in the case of $\theta_{0}=90^{\circ}$ and $180^{\circ}$.

A consequence of MacDonald's theorem, "as $\theta$ increases from 0 to $\pi$, any zero diminishes" (Ref. 5), is that the differences between successive zeros, for the same value of $\theta$, decrease with increasing $\theta$ from 0 to $\pi$.

For $\theta_{0}=165^{\circ}$ the maximum dufference between the successive zeros $1 \mathrm{~s} 1.0909$ and the minimum difference is 1 ; for $\theta_{0}=170^{\circ}$ the maximum difference is 1.0588 
2. The Method of Solution. Schelkunoff $[6, p .514]$ expanded the Legendre unction in a Taylor expansion

$$
P_{n^{\prime}}+z_{n}(x)=P_{n^{\prime}}(x)+2 z_{n^{\prime}}\left[\frac{1}{2} P_{n^{\prime}}(x) \log \frac{1}{2}(1+x)+S_{n^{\prime}}^{\prime}\right]
$$

where (with $\left[n^{\prime}\right]=$ largest integer in $n^{\prime}$ )

$$
S_{n^{\prime}}^{\prime}=\sum_{s=1}^{\left[n^{\prime}\right]} \frac{(-1)^{s}\left(n^{\prime}+s\right)^{\prime}}{(s !)^{2}\left(n^{\prime}-s\right)^{\prime}}\left\{\frac{1}{n^{\prime}+s}+\cdots+\cdots+\frac{1}{n^{\prime}+1}\right\}\left(\frac{1-x}{2}\right)^{s}
$$

Another form for the $S_{n}^{\prime}$ is

$$
S_{n^{\prime}}^{\prime}=(-1)^{\left[n^{\prime}\right]} \sum_{s=0}^{\left[n^{\prime}\right]} \frac{(-1)^{s}(n+s)^{\prime}}{(s !)^{2}(n-s) !}\left\{\frac{1}{s+1}+\cdots+\frac{1}{s+n^{\prime}}\right\}\left(\frac{1+x}{2}\right)^{s}
$$

Differentiating (2.1) with respect to $x$ we obtain

$$
\frac{d P_{n^{\prime}}+Z_{n^{\prime}}(x)}{d x}=\frac{d P_{n}(x)}{d x}+z_{n^{\prime}}\left\{\frac{d P_{n^{\prime}}(x)}{d x} \log \frac{1+x}{2}+\frac{P_{n}(x)}{1+x}+2 \frac{d S_{n^{\prime}}^{\prime}}{d x}\right\}
$$

Setting $x=x_{0}$ and remembermg that $P_{n_{1}}^{\prime}\left(x_{0}\right)=0$ we obtain

$$
z_{n^{\prime}}=\left(\frac{-\frac{d P_{n^{\prime}}(x)}{d x}}{\frac{P_{n^{\prime}}(x)}{1+x}+\frac{d P_{n^{\prime}}(x)}{d x} \log \frac{1+x}{2}+2 \frac{d S_{n^{\prime}}^{\prime}}{d x}}\right)_{x=x_{0}}
$$

where $n_{\imath}=n^{\prime}+z_{n^{\prime}}$

Equation (2.3) is a relationship between Legendre Functions and Associated Legendre Functions and a finite sum $d S_{n^{\prime}}^{\prime} / d x$. To make it numerically useful one must be able to evaluate the functions on the right without having to sum slowly convergent series.

If we consider $n^{\prime}$ either an integer or an odd multiple of $\frac{1}{2}$, we can evaluate the functions on the right of $(23)$ quite easily. Some tables exist for the Legendre Polynomials and their derivatives (Ref. 7 and 8) When $n^{\prime}$ is considered an odd multiple of $\frac{1}{2}$, several methods of obtaining tables are avalable when one utilizes Hall's observation (Ref. 9) that the $\frac{1}{2}$ integer $P$ 's can be expressed in terms of the complete elliptic integrals, $\mathrm{K}$ and $\mathrm{E}$, of modulus $\frac{1}{2}\left(1-x_{0}\right)$.

$$
\begin{aligned}
P_{-\frac{1}{2}}(x) & =(2 / \pi) K \\
P_{\frac{1}{2}}(x) & =(2 / \pi)(2 E-K)
\end{aligned}
$$

(Ref. 9, p. 927)

These functions have been tabulated (Ref. 10). There are many ways to obtain the other $\frac{1}{2}$ integer Legendre and Associated Legendre Functions. One could use the recursion relationship between the Legendre and Associated Legendre Functions. In Section 4 these functions for $x_{0}=\cos 165^{\circ}$ are evaluated, and the existing tables were used as checks.

To improve our results we added a third term to the Taylor expansion (2.1) and thus instead of (2.3) we solved a resulting quadratic equation in $z_{n^{\prime}}$.

Thus, to obtain the zeros of

$$
P_{n_{2}}^{\prime}\left(x_{0}\right)=\left[-\sqrt{1-x^{2}}\left(d P_{n_{2}}(x) / d x\right)\right]_{x=x_{0}}=0
$$


we set $n_{\imath}=n^{\prime}+z_{n^{\prime}}$ and compute two columns (one for $n^{\prime}=j+\frac{1}{2}$ and one for $n^{\prime}=\jmath ; j=1,2,3, \cdots \quad$ ), as shown in Table 2 .

Table 2 is obtained from a Taylor expansion in $z$ which was terminated after three terms, thus committing an error proportional to $z$ cubed. The obvious criteria then is to choose $a_{k}$ if $\left|z_{k}^{\prime \prime}\right|<\left|z_{k}^{\prime}\right|$ or $a_{k}^{\prime}$ If $\left|z_{k}^{\prime \prime}\right|>\left|z_{k}^{\prime}\right|$.

A method of predicting which part of Table 2 should be used, even before the $n_{\imath}$ 's are computed, is avarlable for most of the $n_{i}$ 's for any given angle. We shall proceed to illustrate this for $x_{0}=\cos 165^{\circ}$.

TABLE 2

\begin{tabular}{|c|c|c|}
\hline Zeros $\left(n_{2}\right)$ & $\begin{array}{c}\mathrm{A} \\
\jmath+\frac{1}{2} \pm Z_{j}^{\prime \prime}\end{array}$ & $\begin{array}{c}\mathrm{A}^{\prime} \\
\jmath \pm Z_{z}^{\prime}\end{array}$ \\
\hline$n_{1}$ & $a_{1}$ & $a_{1}^{\prime}$ \\
\hline$n_{2}$ & $a_{2}$ & $a_{2}^{\prime}$ \\
\hline$n_{k}$ & $a_{k}$ & $a_{k}^{\prime}$ \\
\hline
\end{tabular}

where $Z_{1}^{\prime \prime}=Z_{j+\frac{1}{3}} \& Z_{j}^{\prime}=Z_{1}$

In Reference 2 it was seen that the integer parts of the zeros could be determined from the Associated Legendre Functions. The example given in that reference was

$$
\begin{aligned}
& \frac{1}{\sin 165^{\circ}} P_{13}^{\prime}\left(\cos 165^{\circ}\right)=6.50140 \\
& \frac{1}{\sin 165^{\circ}} P_{14}^{\prime}\left(\cos 165^{\circ}\right)=-.86327 \\
& \frac{1}{\sin 165^{\circ}} P_{15}^{\prime}\left(\cos 165^{\circ}\right)=-5.23851
\end{aligned}
$$

and

$$
1.0321>n_{1}>1.0316
$$

From (2.6) we note that a change in sign exists between $\mathrm{P}_{13}^{\prime}\left(\cos 165^{\circ}\right)$ and $P_{14}^{\prime}\left(\cos 165^{\circ}\right)$ but not between $P_{14}^{\prime}\left(\cos 165^{\circ}\right)$ and $P_{15}^{\prime}\left(\cos 165^{\circ}\right)$. Thus, $P_{n_{2}}^{\prime}\left(x_{0}\right) \neq 0$ for $14 \leqq n_{\imath} \leqq 15$. Since by (2.7) $n_{1} \approx 1.032$ and since the largest difference is 1.0909 , then each $n_{\imath}$ for $1 \leqq i \leqq 13$ has the number $i$ to the left of the decimal point. For $14 \leqq i \leqq N$, where $N$ is at least 23 , the $n_{\imath}$ has the number $(i+1)$ to the left of the decimal point.

Since we have observed maximum and minimum differences for $\cos 165^{\circ}$ 
(1.0909 and 1) we can obtain more information from the fact that no change in sign occurs between $P_{14}^{\prime}\left(\cos 165^{\circ}\right)$ and $P_{15}^{\prime}\left(\cos 165^{\circ}\right)$; namely,

$$
\begin{aligned}
& 16.1818>n_{15}>16,\left|z_{15}^{\prime}\right|<.1818 \\
& 15.0909>n_{14}>15,\left|z_{14}^{\prime}\right|<.0909 \\
& 14>n_{13}>13.9091,\left|z_{13}^{\prime}\right|<.0909 \\
& 13>n_{12}>12.8182,\left|z_{12}^{\prime}\right|<.1818
\end{aligned}
$$

and also, from (2.7)

$$
\begin{aligned}
& 1.0321>n_{1}>1.0316 \\
& 2.1230>n_{2}>2.0316 \\
& 3.2139>n_{3}>3.0316
\end{aligned}
$$

By (2 9), for at least the first three zeros, column $\mathrm{A}^{\prime}$ of Table 2 is better than column A. By (2.8), for $n_{12}, n_{13}, n_{14}$, and $n_{15}$, again column $\mathrm{A}^{\prime}$ is better. The same sort of reasoning can be applied to the $P_{2}^{\prime}+\frac{1}{2}(x)$ to tell when column A of Table 2 should be used.

Since there is no change in sign between $P_{7.5}^{\prime}\left(x_{0}\right)$ and $P_{85}^{\prime}\left(x_{0}\right)$ and no change in sign between $P_{195}^{\prime}\left(x_{0}\right)$ and $P_{205}^{\prime}\left(x_{0}\right)$, it can be concluded that

$$
\begin{aligned}
& 9.6818>n_{9}>9.5,8.5909>n_{8}>8.5, \\
& 7.5>n_{7}>7.4091,6.5>n_{6}>6.3182
\end{aligned}
$$

and

$$
\begin{aligned}
& 21.6818>n_{20}>21.5,20.5909>n_{19}>20.5, \\
& 19.5>n_{18}>19.4091,18.5>n_{17}>18.3182 .
\end{aligned}
$$

and thus for the zeros indicated in (2 10) and (2.11), the results of column $\mathrm{A}$ in Table 2 should be used.

Then there will be very few cases where one should average between the tabulated data of columns $A$ and $A^{\prime}$ in Table 2 In Section 4 in our numerical example we shall follow this type of reasoning and select either the value in column $\mathrm{A}$ or that in column $\mathrm{A}^{\prime}$. It could be pointed out that instead of using a table with two columns and thus creating a scientific selection process, one could use either column $\mathrm{A}$ or $\mathrm{A}^{\prime}$ and add more terms to the Taylor expansion Not only would this add more work than the suggested method (and also create places of increased error) but also would remove redundancy from the system. Since these computations were carried out on hand calculating machines, whenever we have a choice between two methods of almost equal over-all accuracy we choose the method with the most redundancy, a valuable means of checking computations. In this method we always have two values of $n_{\imath}$ although one of these values is discarded in the final result The two values serve as a check inasmuch as we would immediately be alerted if the two values were very different.

The integrals $\int_{1}^{x_{0}}\left[P_{n_{2}}(x)\right]^{2} d x, \int_{1}^{x_{0}}\left[P_{n_{2}}^{\prime}(x)\right]^{2} d x$, and $\int_{1}^{x_{0}}\left[P_{m_{2}}^{\prime}(x)\right]^{2} d x$ have been 
of much interest in our electromagnetic scattering work (Ref. 11). In Reference 11 , it was shown that

$$
\int_{1}^{x_{0}}\left[P_{n_{1}}^{\prime}(x)\right]^{2} d x=n_{\imath}\left(n_{\imath}+1\right) \int_{1}^{x_{0}}\left[P_{n_{1}}(x)\right]^{2} d x
$$

where the $n_{2}$ are the zeros of $P_{n_{2}}^{\prime}\left(x_{0}\right)=0$. It was also shown that

$$
\int_{1}^{x_{0}}\left[P_{n_{\imath}}(x)\right]^{2} d x=\frac{-\left(1-x_{0}^{2}\right)}{2 n_{z}+1} P_{n_{\imath}}\left(x_{0}\right)\left[\frac{\partial^{2} P_{y}(x)}{\partial x \partial y}\right]_{y=n_{3}, x=x_{0}}
$$

In addition it was shown that when the $m_{\imath}$ are zeros of $\left[d P_{m_{\imath}}^{\prime}(x) / d x\right]_{x=x_{0}}=0$ we obtain

$$
\begin{aligned}
\int_{x_{0}}^{1}\left[P_{m_{\imath}}^{\prime}(x)\right]^{2} d x=m_{\imath}\left(m_{\imath}+1\right)\left(x_{0}^{2}-1\right) P_{m_{\imath}}\left(x_{0}\right) \\
\cdot\left[\frac{P_{m_{\imath}}(x)}{x}+\frac{m_{\imath}\left(m_{\imath}+1\right)}{x\left(2 m_{\imath}+1\right)} \frac{\partial P_{y}(x)}{\partial y}+\frac{1}{2 m_{\imath}+1} \frac{\partial^{2} P_{y}(x)}{\partial x \partial y}\right]_{y=m_{\imath}, x=x_{0}}
\end{aligned}
$$

With respect to these integrals we can employ the methods described in this section to the right hand members of $(213)$ and (2.14). In Section 3 the requisite algebra for (2.13) is shown and in Section 4 the results of its application to the case $x_{0}=\cos 165^{\circ}$ are given.

One might observe that the algebra required to put (2.14) in a similar computational form would be no more difficult, although it would be more lengthy.

3. The Computational Forms. In Section 2 the method of approach used in finding the $a_{k}$ and $a_{k}^{\prime}$ of Table 2 was described, in this section we display the details of the computational method

For column $A^{\prime}$ of Table 2 we start with

$$
P_{\jmath+z_{j}^{\prime}}(x)=\left[P_{y}(x)+z_{\jmath}^{\prime} \frac{\partial P_{y}(x)}{\partial y}+\frac{1}{2} z_{3}^{\prime 2} \frac{\partial^{2} P_{y}(x)}{\partial y^{2}}\right]_{y=\text { 。 }}
$$

and for column $\mathrm{A}$ of the table we start with

$$
P_{\jmath}+1+z_{,}^{\prime \prime}(x)=\left[P_{y}(x)+z_{j}^{\prime \prime} \frac{\partial P_{y}(x)}{\partial y}+\frac{1}{2} z_{3}^{\prime \prime 2} \frac{\partial^{2} P_{y}(x)}{\partial y^{2}}\right]_{y=1+\frac{1}{2}}
$$

Differentiating these equations with respect to $x$, evaluating at $x=x_{0}$, and under the stipulation that $n_{i}=j+z_{1}^{\prime}$ in (3.1a) and $n_{\imath}=\jmath+\frac{1}{2}+z_{1}^{\prime \prime}$ in (3.1b), we obtain the quadratic equations referred to in Section 2. Solving them for $z_{3}$ we obtain, in the first case

$$
\begin{aligned}
z_{,}^{\prime}= & {\left[\left\{\frac{\partial^{3} P_{y}(x)}{\partial x \partial y^{2}}\right\}^{-1}\right.} \\
& \cdot\left\{-\frac{\partial^{2} P_{y}(x)}{\partial x \partial y} \pm \sqrt{\left.\left(\frac{\partial^{2} P_{y}(x)}{\partial x \partial y}\right)^{2}-2\left(\frac{\partial P_{y}(x)}{\partial x}\right)\left(\frac{\partial^{3} P_{y}(x)}{\partial x \partial y^{2}}\right)\right\}}\right]_{y=1, x=x_{0}}
\end{aligned}
$$


and in the second case

$$
\begin{aligned}
& z_{3}^{\prime \prime}=\left[\left\{\frac{\partial^{3} P_{y}(x)}{\partial x \partial y^{2}}\right\}^{-1}\right. \\
& \cdot\left\{-\frac{\partial^{2} P_{y}(x)}{\partial x \partial y} \pm \sqrt{\left(\frac{\partial^{2} P_{y}(x)}{\partial x \partial y}\right)^{2}-2\left(\frac{\partial P_{y}(x)}{\partial x}\right)\left(\frac{\partial^{3} P_{y}(x)}{\partial x \partial y^{2}}\right)}\right\}_{y-\jmath+\frac{1}{2}, x=x_{0}}
\end{aligned}
$$

The expressions that were computed in order to find the $z$, associated with a given $j$ or $j+\frac{1}{2}$ are those shown in (3.2a) and (32b). However, before we comment on them, let us turn our attention to the integral, referred to in Section 2 ,

$$
\int_{x_{0}}^{1}\left[P_{n_{\imath}}(x)\right]^{2} d x=\frac{1-x_{0}^{2}}{2 n_{\imath}+1} P_{n_{1}}\left(x_{0}\right)\left[\frac{\partial^{2} P_{y}(x)}{\partial x \partial y}\right]_{y=n_{\imath}, x=x_{0}}
$$

In order to find both the $n_{\imath}$ 's and the values of these integrals we observe that in addition to the forms required in the right hand members of (3.2a) and (3.2b) we will need $P_{n_{i}}\left(x_{0}\right)$ and $\left[\partial^{2} P_{y}(x) / \partial x \partial y\right]_{y=n_{\imath}}, x=x_{0}$. After the $z$,'s have been obtained, the $P_{n_{2}}\left(x_{0}\right)$ are determined through (3.1a) and (3.1b). As for $\left[\partial^{2} P_{y}(x) / \partial x \partial y\right]_{y=n_{z}, x=x_{0}}$, we consider

$$
\left.\frac{\partial P_{y}(x)}{\partial y}\right|_{y=n,+z,}=\left[\frac{\partial P_{y}(x)}{\partial y}+z_{j} \frac{\partial^{2} P_{y}(x)}{\partial y^{2}}+\frac{1}{2} z_{j}^{2} \frac{\partial^{3} P_{y}(x)}{\partial y^{3}}+\cdots\right]_{y=n_{j}}
$$

and

$$
P_{n,}+z_{j}(x)=\left[P y(x)+z_{3} \frac{\partial P_{y}(x)}{\partial y}+\frac{1}{2} z_{2}^{2} \frac{\partial^{2} P_{y}(x)}{\partial y^{2}}+\cdots\right]_{y=n_{3}}
$$

where $n_{\jmath}=j$ in the one case and $n,=j+\frac{1}{2}$ in the other.

Combining these two equations, differentiating with respect to $x$, and then solving for $\left[\partial^{2} P_{y}(x) / \partial x \partial y\right]_{y=n}+\varepsilon$, we obtain, with the boundary condition $P_{n_{1}}^{\prime}\left(x_{0}\right)=0$ which is equivalent to $\partial P_{n_{3}}(x) /\left.\partial x\right|_{x=x_{0}}=0$,

$$
\left.\frac{\partial^{2} P_{y}(x)}{\partial x \partial y}\right|_{y=n,+z_{2}, x=x_{0}} \cong\left\{-\frac{1}{z} \frac{\partial P_{n_{y}}(x)}{\partial x}+\frac{z}{2} \frac{\partial^{3} P_{y}(x)}{\partial x \partial y^{2}}\right\}_{y=n_{1}, x=x_{0}}
$$

Now examination of (3.1a), (3.1b), (3.2a), (3.2b) and (35) shows what quantities require computation. For both the $j+z^{\prime}$, and $j+\frac{1}{2}+z_{1}^{\prime \prime}$ methods, we start with the form

$$
\begin{gathered}
P_{y}(x)=\frac{\sin y \pi}{\pi} \sum_{s=0}^{\infty} \frac{(-1)^{s}(y+s) !}{(s !)^{2}(y-s)^{\prime}} \\
\cdot\left\{\log \frac{1+x}{2}+\psi(y+s)+\psi(y-s)-2 \psi(s)\right\}\left(\frac{1+x}{2}\right)^{\prime} \\
\quad+\cos y \pi \sum_{s=0}^{\infty} \frac{(-1)^{s}(y+s) !}{\left(s^{\prime}\right)^{2}(y-s) !}\left(\frac{1+x}{2}\right)^{s}
\end{gathered}
$$

which is transformed by the use of the relation

$$
\psi(-z)=\psi(z-1)+\pi \cot \pi z
$$


into

$$
\begin{aligned}
& P_{y}(x)=\frac{\sin y \pi}{\pi} \sum_{s=0}^{\jmath} \frac{(-1)^{s}(y+s) !}{(s !)^{2}(y-s) !} \\
& \cdot\left\{\log \frac{1+x}{2}+\psi(y+s)+\psi(y-s)-2 \psi(s)\right\}\left(\frac{1+x}{2}\right)^{s} \\
& \quad+\cos y \pi \sum_{s=0}^{\jmath} \frac{(-1)^{s}(y+s) !}{(s !)^{2}(y-s) !}\left(\frac{1+x}{2}\right)^{s} \\
& \quad+\frac{\sin y \pi}{\pi} \sum_{s^{\prime}=0}^{\infty} \frac{(-1)^{s^{\prime}+\jmath+1}}{\left[\left(s^{\prime}+\jmath+1\right)\right]^{2}} \frac{\left(y+s^{\prime}+\jmath+1\right) !}{\left(y-j-1-s^{\prime}\right) !} \\
& \cdot\left\{\log \frac{1+x}{2}+\psi\left(y+s^{\prime}+\jmath+1\right)+\psi\left(\jmath+s^{\prime}-y\right)-2 \psi\left(s^{\prime}+\jmath+1\right)\right\} \\
& \quad \cdot\left(\frac{1+x}{2}\right)^{s^{\prime}+\jmath+1}
\end{aligned}
$$

Starting with (3.8) each of the derivatives required is determined and approximated by taking a finite number of terms in the corresponding series. The number of terms one would use would depend upon the number of significant digits required. It was found that the first $(j+1)$ terms of each series would would give sufficient accuracy for our purposes. For example in finding $\left[\partial P_{y}(x) / \partial y\right]_{y=\jmath}+, x=x_{0}$, when $\jmath=1$ the error committed by restricting the sum to the first $(\jmath+1)$ terms is less than .009 while for $\jmath=3$ the error is less than $3 \times 10^{-6}$.

With forms determined for the derivatives, the equations (3.2a) and (3.2b) could then be employed to determine the values of $z_{3}^{\prime \prime}$ and $z_{3}^{\prime}$. Then the values of the zeros are determined by

$$
n_{\iota}=z_{2}^{\prime}+\jmath
$$

$$
\text { where } \begin{aligned}
i & =\jmath \text { for } 1 \leqq i \leqq 13 \\
\imath & =\jmath-1 \text { for } 14 \leqq \imath \leqq 23
\end{aligned}
$$

and

$$
n_{2}=\jmath+\frac{1}{2}+z_{\jmath}^{\prime \prime}
$$

To determine the values of the integrals both the derivatives and the Legendre and the Associated Legendre Functions of integer degree are required. These Legendre and Associated Legendre Functions of integer degree were expressed in polynomial form so that therr values could be determined by substitution. With these determined, the integrals are placed in computational form (see (3.5) and (3.3))

The results that one obtains when this scheme is used are illustrated by the example which is discussed in the following section

4. Results for $x_{0}=\cos 165^{\circ}$. The values for Legendre and Associated Legendre Functions of integer and half-integer degree are tabulated in Table 3.

Table 4 contains the results of our computation. 
TABLE 3

Values of Legendre Functions for $x_{0}=\cos 165$

\begin{tabular}{|c|c|c|c|c|c|c|c|c|c|c|}
\hline \multirow{2}{*}{$\frac{y}{1}$} & \multicolumn{2}{|c|}{$P_{y}\left(x_{0}\right)$} & & \multicolumn{2}{|c|}{$-P_{y}^{\prime}\left(x_{0}\right) / \sqrt{1-x_{0}^{2}}$} & \multirow{2}{*}{$\frac{y}{-5}$} & \multicolumn{2}{|c|}{$P_{\boldsymbol{y}}\left(x_{0}\right)$} & \multicolumn{2}{|c|}{$-P_{\nu}^{\prime}\left(x_{0}\right) / \sqrt{1-x_{0}^{2}}$} \\
\hline & -96592 & 58263 & & +100 & & & 2.1854 & 43649 & & \\
\hline 2 & +.89951 & 90528 & & -28977 & 77479 & 0 & -88034 & 27550 & & \\
\hline 3 & -80416 & 39229 & & +5.4975 & 95264 & 15 & .40531 & 3187 & -10946 & 2606 \\
\hline 4 & +68469 & 54388 & & -85269 & 24940 & 25 & -098198 & 308 & & 5587 \\
\hline 5 & -54712 & 58747 & & +11659 & 85421 & 35 & -12690 & 551 & -11. & 45 \\
\hline 6 & 39830 & 59910 & & & 30956 & 45 & & 8791 & & 31 \\
\hline 7 & .24554 & 10452 & & & 83210 & 55 & -.41 & & -8 & 626 \\
\hline 8 & +09618 & 43272 & & -18228 & 42524 & 65 & 487 & 2435 & & 24 \\
\hline 9 & +.04276 & 78470 & 9 & +18.472 & 96566 & 75 & -52104 & 2564 & & 28 \\
\hline 10 & -16505 & 59738 & & -17 & 83615 & 8.5 & & 732 & -2 & 56 \\
\hline 11 & - 26548 & 99921 & & & 79021 & 95 & -48 & & & 83 \\
\hline 12 & -34021 & 56675 & & -11 & 56633 & 105 & .41 & 3951 & -12 & 70 \\
\hline 13 & +38689 & 98145 & & +65013 & 98521 & 115 & -.32992 & 1717 & 16.692 & 590 \\
\hline 14 & -40482 & 44602 & & -86327 & 13353 & 125 & 22922 & 4586 & -20247 & 8253 \\
\hline 15 & +39488 & 56155 & & -5.2385 & 10824 & 135 & -.12094 & 3791 & 22652 & 4294 \\
\hline 16 & -35949 & 81090 & & +11378 & 18274 & 145 & & 729 & -23634 & 2515 \\
\hline 17 & +30241 & 35834 & & $-17 \quad 101$ & 94842 & & & 636 & & 611 \\
\hline 18 & -22846 & 39061 & & & 65816 & & -.18 & 162 & & $97:$ \\
\hline 19 & +.14324 & 66015 & & -25555 & 11295 & 175 & & 9737 & & 05 \\
\hline 20 & -.05277 & 21934 & 5 & +27549 & 27562 & 185 & -.30642 & 7865 & -11.598 & 345 \\
\hline & & & & & & & 33582 & 976 & 5.2278 & 0 \\
\hline & & & & & & 20.5 & -341 & 94 & 18349 & \\
\hline
\end{tabular}

TABLE 4

Results for the case $x_{0}=\cos 165^{\circ}$

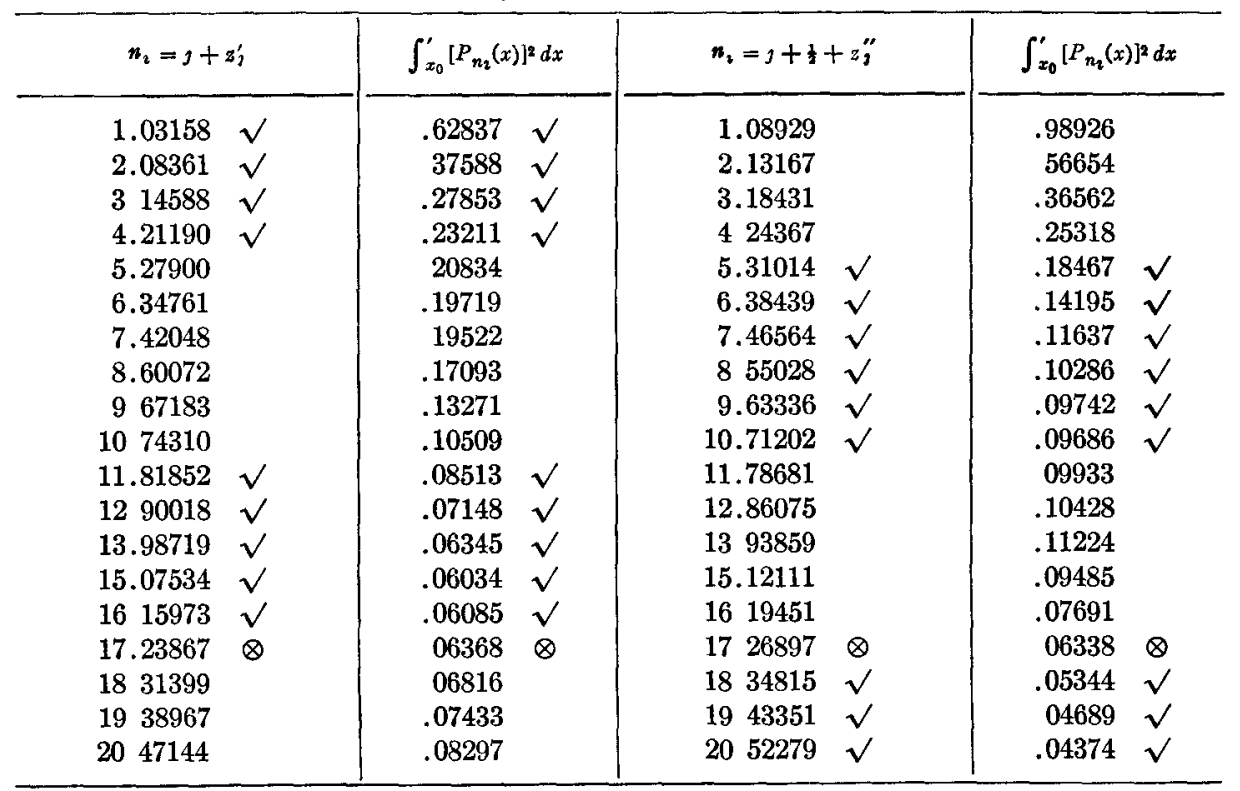

$\checkmark$ indicates preferred value (according to criterion of Section $* 2$ ).

$\otimes$ indicates need for averaging (according to criterion of Section $* 2$ ). 
In conclusion it should be pointed out that no effort has been made to smooth out results as the major virtue of this method is the convenience and speed of computation.

The series for the Legendre Functions involved are very slowly convergent and any effort made to "reinforce" unreliable entries in Table 4 would imply the knowledge of a correct entry (the calculation of which we wish to by-pass in obtaining answers to physical problems).

\section{REFERENCES}

1 P A Carrus and C G Treuensfels, "Tables of Roots and Incomplete Integrals of Associated Legendre Functions of Fractional Orders "Journal of Mathematics and Physics, Vol XXIX No 4, January 1951

2. K. M Siegel, D. M Brown, H E Hunter, H A Alperin, C. W. Quillen, "The Zeros of the Associated Legendre Functions $P_{n}^{m}\left(\mu^{\prime}\right)$ of Non-integral Degree," Willow Run Research Center, University of Michıgan, April, 1951

3. E W Hobson, "The Theory of Spherical and Ellipsoidal Harmonics, Cambridge University Press, 1931

4 W. Magnus \& F Oberhettinger, "Formulas and Theorems for the Special Functions of Mathematical Physics," Chelsea Publishing Company, New York, 1949.

5. H. M MacDonald, "Zeros of Spherical Harmonic $P_{n}^{m}(\mu)$ Considered as a Function of $n$," Proceedings of London Mathematical Society, No I, Vol. XXXI (1900).

6 S A Schelkunoff, "Theory of Antennas of Arbitrary Size and Shape," Proceedings of IRE, Vol 29, 1941

7 S D Hamren, "Scattering from Spheres," Institute of Engmeering Research, University of California, June, 1950

8 "Tables of Functions of First and Second Partial Derivatives of Legendre Polynomials," Engineerıng Research Institute, University of Michigan, November, 1951

9 R N HALL, "The Application of Non-integral Legendre Functions to Potential Problems," Journal of Applied Physics, Vol 20, October 1949

10. Oberhettinger and Magnus, "Anwendung Der Elliptischen Functionen in Physik und Technık," Julius Springer, Berlin, 1949.

11. K. M. Sieger and H. Alperin, "Scattering by a Semi-infinite Cone," Willow Run Research Center, University of Michigan, UMM-87, to be published.

12. S. A. Schelkunoff, "Electromagnetze Waves," D Van Nostrand, Inc New York, 1943

13. JAhNKe \& Emde, "Tables of Functions with Formulae and Curves," Dover Publications, New York, 1945

Willow Run Research Center of University of Michigan

(Recelved February 25, 1952) 